NOTES ON THE ACTION OF ATROPINE, HYOSCYAMINE, HYOSCINE, SCOPOLAMINE, DUBOISINE, AND DATU. RINE

By W. WEBSTER, M.D., C.M., Anaesthetist to the Winnipeg General Hospital, Lecturer in Anaesthetics in the Manitoba Medical College.

From the Physiological Laboratory of the University of Manitoba

Communicated by Professor Swale Vincent, M.B., D.Sc.

(Received March 8th, 1908)

The primary object of the present research was to test the efficiency of atropine as a restorative in poisoning by chloroform or other anaesthetic, or as a precautionary measure before its administration. The use of atropine in one or other of these ways has been frequently advocated. ${ }^{1}$

At the outset of my series of experiments a somewhat striking discrepancy was noticed between the statements in the text-books and the results actually obtained, even as regards the most readily observable effects upon the heart and blood vessels; thus it was deemed advisable to perform an extensive series of experiments devoted to the physiological effects of atropine and its allies upon the heart, respiration and circulation, apart altogether from the question of benefit or otherwise in the administration of anaesthetics.

Atropine and the allied drugs mentioned in the title are generally supposed to be isomeric with each other, or very closely allied. There is a close relationship between these substances as regards not only their chemical structure but also their physiological action, and as far as my experiments are concerned, it is impossible to detect any difference between them. In regard both to their general physiological effects and to the question of the rapidly induced tolerance or immunity described later, all these drugs may be considered as

1. Dixon, Manual of Pbarmacology, p. 79, London, 1906, 
identical. ${ }^{1}$ My experiments, however, have been restricted to what we may call gross effects upon the heart, circulation and respiration. The effects on the central nervous system, nerve endings, secretion, and excretion have not been specially examined in the present investigation, and it is, of course, possible that in some of these spheres of action there may be individual differences between the different drugs.

The question of the value of adrenalin as a restorative to the circulatory system will be incidentally discussed in the body of the paper.

I have performed in all more than fifty experiments-two on cats, the rest on dogs. Chloroform, ether, or the A.C.E. mixture were the anaesthetics used. In some few cases curari has been used in addition. The blood pressure has been taken from the carotid artery, and the injections made into the saphenous or femoral vein. A glass plethysmograph was used for recording changes in the volume of the limb, and for similar changes in the intestinal wall an air oncometer was used, each of these being connected with a piston recorder. The method described by Oliver and Schaefer ${ }^{2}$ was employed for recording the effects upon the heart. A hook is caught in the epicardium of the auricle, and another in that of the ventricle. From these threads pass over pulleys moving on a horizontal axis; the threads then pass vertically downwards to be attached to long elastic levers of steel. To the ends of the levers writing points are attached.

\section{Physiological Effects}

One of the most familiar, and at the same time most striking, actions of atropine is paralysis of the peripheral terminations of the vagus in the heart. It would naturally be expected that this effect, like section of the vagi, since it cuts off the tonic inhibitory influence

I. For information on the chemistry of these substances see Schmiedeberg, Pbarmakologie, 1906; Tomasini, Atti dell. R. Accad. dell. Scienz. Med., Palermo, Ann. 1896; O. Hesse, Leibig's Annalen; Vól. CCLXXVII, 304, 308 ; O. Hesse, in Apotbeker Zeitung, 1895 ; Raehlmann, Semaine Medicale, 1893 ; Bokenham, B.M.F., Vol. II, p. 597, 1894; T. R. Pooley, Car. Lancet, Jan., 1895; Sharp, Practitioner, Jan., 1894 ; Pavloff, St. Petersberg Med. Cbi. Diss., 1889-1890; Year Book of Pbarmacy, 1880, 188t, 1882, 1885, 1892, 1893, 1894; Pbarm. Fourn., 1878.

2. Fourn. of Pbysiol., Vol. XVIII, p. 256, 1895: 
of the nerve centre, would exercise an augmentor effect upon the heart, and raise the blood pressure. This is, in fact, usually stated to be the case. Thus Dixon ${ }^{1}$ says : 'In mammals small injections of atropine produce the same result : this paralysis of the peripheral vagal terminals, like section of the vagi, cuts off the tonic inhibitory influence of the centre, and the heart is quickened. The increased rate will naturally only occur in those animals in which there is some tonic central effect. Thus the quickening is decided in dogs, and little in cats, whilst in man it varies with the age and disposition, but is usually greatest between the ages of twenty-five and forty. In children under two months atropine causes no quickening, and it has also little effect in old age.' This author also suggests that atropine may directly stimulate cardiac muscle, and refers to a vaso constriction as a result of the action of the drug on the medulla. ' Blood pressure rises mainly as a result of this vaso constriction . . . the pressure also tends to rise on account of the quickened heart . . . . constriction of vessels is pronounced only in the splanchnic area.' Schmiedeberg ${ }^{2}$ likewise states that atropine, in men and dogs, quickens the heart and raises blood pressure, but admits that a subsequent effect is paralysis of the heart. ${ }^{3}$ Sollmann ${ }^{4}$ states that the blood pressure is scarcely altered, but there may be a slight rise from stimulation of the vasomotor centre; this stimulation is always slight, and may be entirely absent. It is replaced by vasomotor depression rather early. Large doses depress the vasomotor centre profoundly, so that the pressure falls very low while the heart is still beating. Still larger doses paralyse the heart muscle as well. Sollmann is the only author I am acquainted with who mentions this lowering of blood pressure at all. Thus Dixon states that there is a rise of blood pressure owing to vaso constriction; Sollmann that there is at first a slight rise owing to vaso constriction, and later a pronounced fall owing to vaso dilatation. But my own experiments

\footnotetext{
I. Op. cit.

2. Op. cit.

3. This secondary effect is also mentioned by Pouchet, Leçons de Pbarmacodynamie, Paris, 1901.

4. Sollmann, Text-book of Pbarmacology, Philadelphia, igo6.
} 
furnish no evidence of any action whatever on the vasomotor system. I have been unable to find any original papers giving details of experiments upon animals, with tracings of the blood pressure. ${ }^{1}$

In my series of more than fifty experiments on dogs $I$ bave never observed any rise of blood pressure upon the injection of atropine into the circulation. ${ }^{2}$

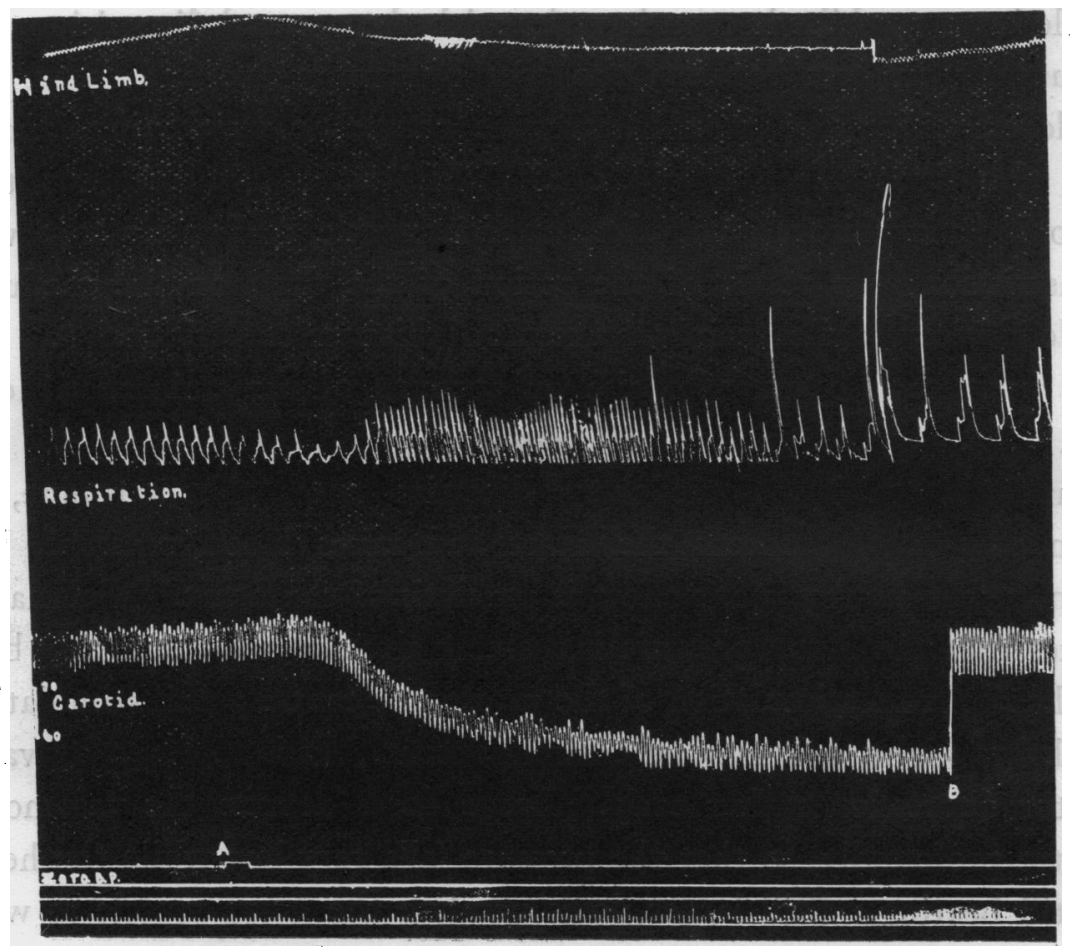

FigURE 1.-Dog, 10.5 kg. Chloroform. No anaesthetic had been given for half an hour before the injection, at A, of 0.4 of a gram of atropine. The animal had previously received, in increasing doses, commencing with 0.7 of a mg. of hyoscine, $50 \mathrm{mg}$. of hyoscine and a 0.5 of a gram of atropine. The blood pressure falls and returns to normal in 7.5 minutes. At B, the kymograph was'stopped for 5 minutes. Respiration was first quickened, then, as the blood pressure returns to the normal, it is markedly slower but deep. The limb follows passively the blood pressure. Scale, half.

I. The literature, however, to which I have access is limited.

2. Professor Vincent informs me that so far as his memory serves him he has never seen a rise of blood pressure on the administration of atropine. 
This applies to the allied drugs mentioned in the introduction. The drugs have been tested under very varying conditions as to dosage, amount of fluid injected, temperature of fluid injected, and rate of injection. In all cases, when any effect whatever has been produced, this has been in the direction of a fall of blood pressure. In small doses this is slight and transient $;^{1}$ in large doses marked and long continued-sometimes for an hour. In some cases, however, even after large doses, recovery is fairly rapid. (See Fig. I.)

In many cases, it is true, the tracings show a slight preliminary rise of blood pressure. This, generally followed by a much more pronounced and significant fall, is, I have convinced myself, simply due to the injection of the fluid in which the drug is dissolved, since an injection of an equal quantity of normal saline solution has been always found to induce a rise of pressure similar in character, and of equal magnitude. (Fig. 2.)

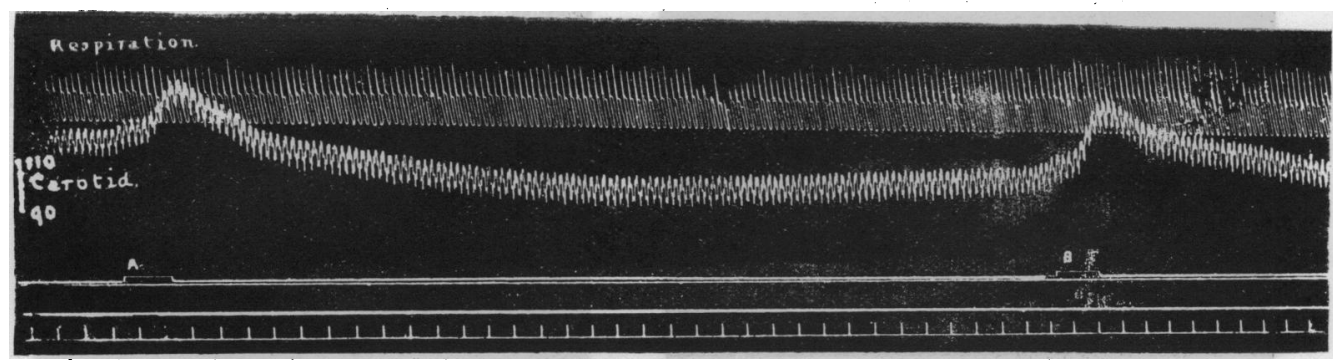

FIgURE 2.-Dog, $5 \cdot 3 \mathrm{~kg}$. A. C. E. At A, 0.5 of a mg. of atropine was given in 10 c.c. saline solution. At B, IO c.c. saline solution only was injected. The same initial rise of blood pressure occurs in both instances. Time intervals, 5 secs.

Of course, the effect of atropine on the blood pressure has almost always been recorded in animals already under the effects of other

I. A possible criticism of my results would be that doses small enough had not been tried, and that the initial increased rate of heart beat and concomitant rise in blood pressure, whether due to this or to vaso constriction, had been overlooked. Every precaution has been taken to avoid this error. No dose, however small, has in my dogs produced the slightest rise of blood pressure. In many cases so small was the amount of drug injected that no effect whatever was produced other than that due to the fluid administered, which point has been very frequently tested by control injections of the same quantity of normal saline solution. 
drugs. Thus the animal has been under the effects of chloroform, ether, or A.C.E. mixture when the first dose of atropine, hyoscine, etc., has been injected, and in the experiment in which records of auricle and ventricle were taken, curari was administered in addition. ${ }^{1}$ On the other hand, many of the larger doses have been given when the animal has not had any anaesthetic for the previous half hour, the atropine already used sufficing to maintain an unconscious condition.

Effect upon the Heart.-Contrarily to the usually accepted view, it has been found that in dogs atropine has only at most a very slight and temporary effect in the direction of augmentation of the heart beats ; the chief effect is a diminution in the extent of movement as revealed by the heart levers. (See Figs. 3 and 4.)

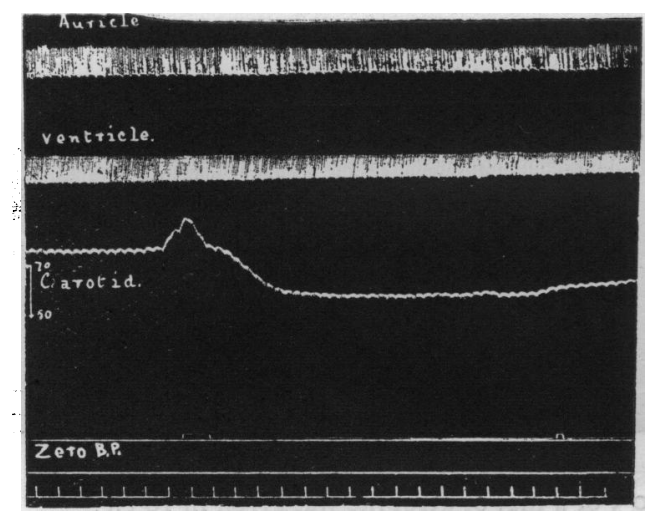

FIG. 3

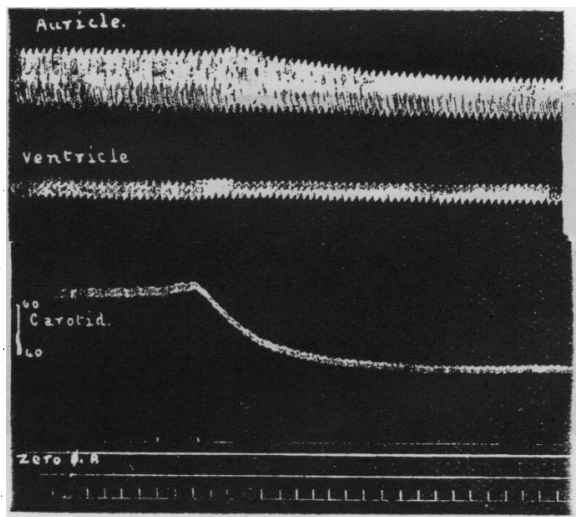

FIG. 4

FIGURE 3.-Dog, 5.2 kg. Ether. I mg. of atropine has the usual effect on carotid pressure. There is slight diminution in extent of movement of auricle and ventricle. Time intervals, 5 secs.

Figure 4.-Dog, II $5 \mathrm{~kg}$. Ether, curari ; artificial respiration. Atropine, I mg. Very slight temporary increase of frequency of heart beat, followed by diminution in extent of movement, as shown also in Figure 3. Time intervals, 5 secs.

i. In one experiment I have reduced this objection to a minimum by employing only just sufficient anaesthetic for the carrying out of the preliminary surgical proceedings. The animal was then allowed to recover from anaesthesia, and atropine in a small dose injected into a vein. The effect in this case, as usual, was a fall and not a rise of blood pressure, and the heart was weakened. (See Fig. 10.) 
Mode of Action of the Drugs.-The levers of the piston recorders connected with the limb plethysmograph and the intestinal oncometer always fall on the injection of the drug. The limb and intestinal tracings, in fact, passively follow that of the blood pressure, and frequently show the same preliminary rise as does the blood pressure. (Figs. 5 and 6.)

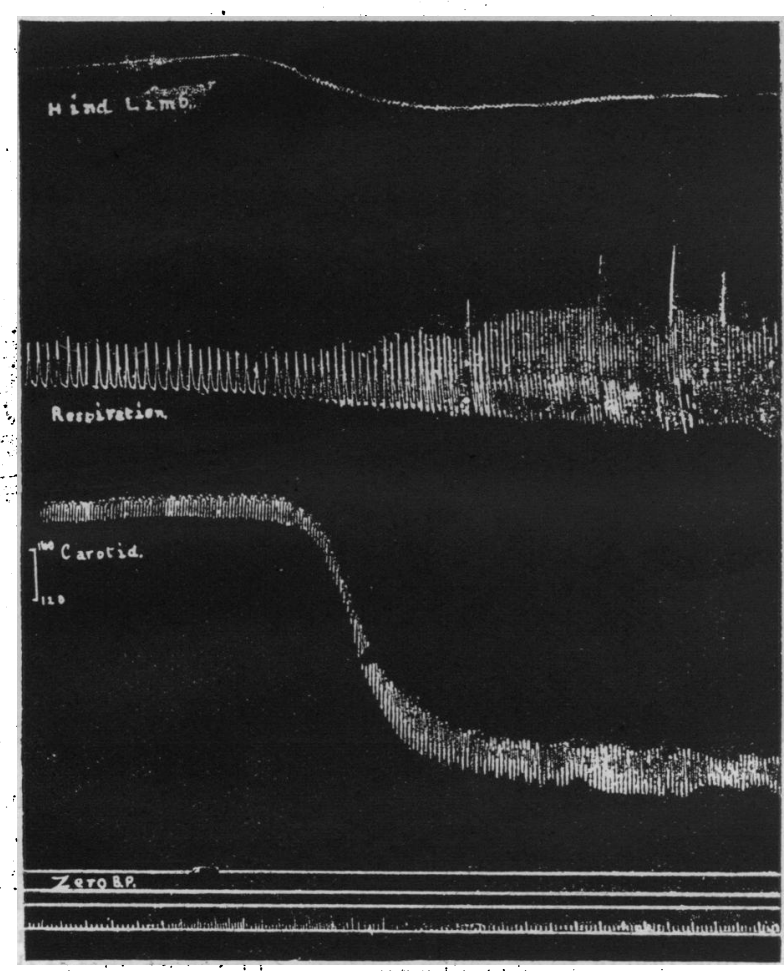

Figure 5.-Dog, $10.5 \mathrm{~kg}$. Chloroform, atropine, 0.2 of a gram. The animal had previously been given increasing doses of hyoscine and atropine. The blood pressure was markedly lowered but the animal recovered. The heart was slowed, respiration was increased in frequency and depth and rendered more irregular. The volume of the limb follows the blood pressure. The heart beat is less frequent and more irregular, as indicated by the movements of the mercury in the manometer. The slowing of the heart beat is shown by the increased excursions of the mercury, which are shown most distinctly at the lowest part of the curve. Such increased excursions are frequently misinterpreted as meaning increased force of heart beat.

This must be interpreted as meaning that owing to enfeebled heart's action blood is drained from both the somatic and splanchnic 
areas; there cannot be active vaso constriction, or there would be a rise of blood pressure, and we have seen that there is never any evidence of vaso dilatation.

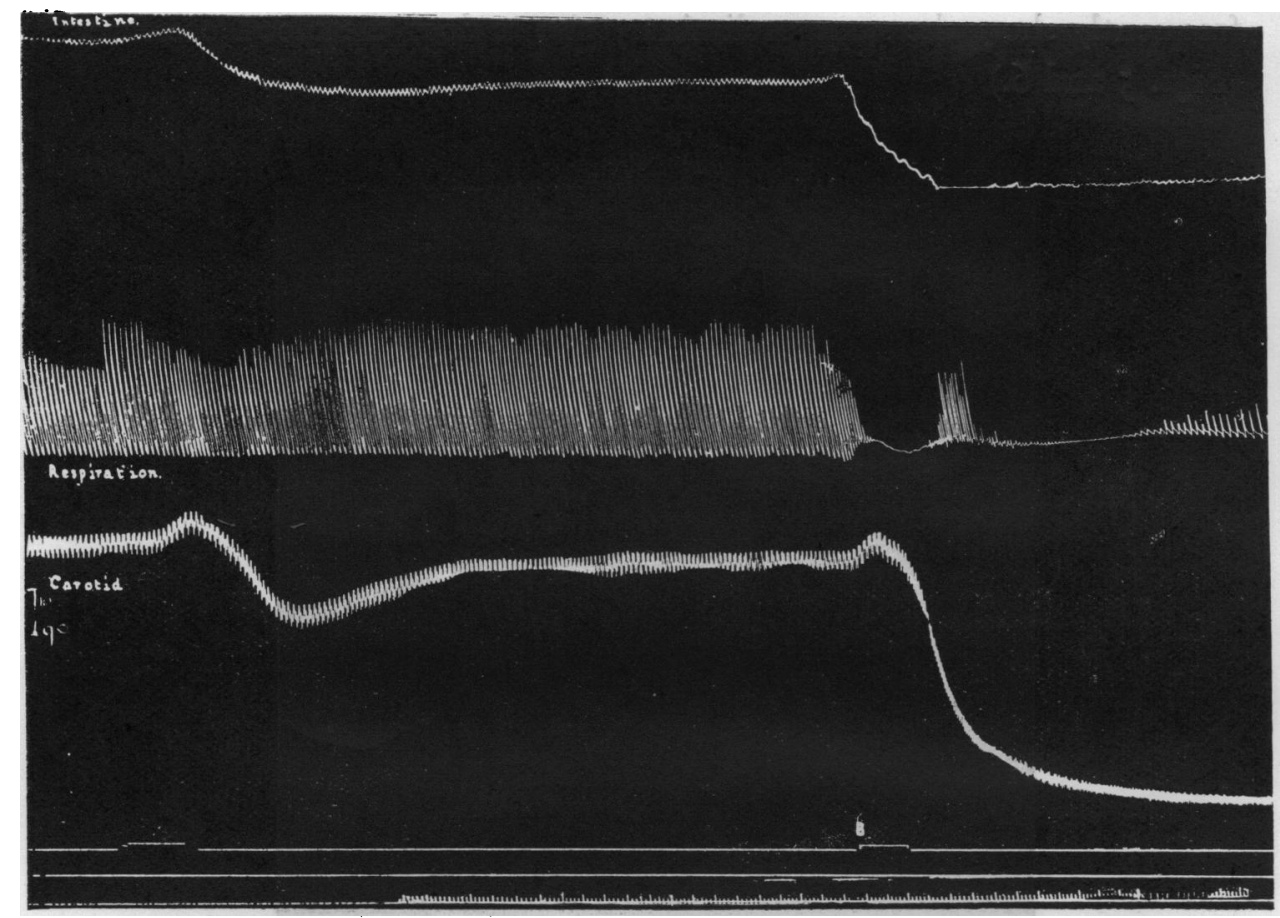

FIGURE 6. - Dog, $7 \mathrm{~kg}$. At A, $8 \mathrm{mg}$. of atropine is administered in 10 c.c. saline solution. The animal has already had $7.5 \mathrm{mg}$, of atropine in divided doses. At B, $20 \mathrm{mg}$. of atropine, in 10 c.c. saline solution, proved fatal in to minutes. Intestinal volume follows the blood pressure after both doses. The slight preliminary rise of blood pressure is usually obtained and is due to the quantity of fluid injected, as it can be invariably reproduced by the injection of $\mathrm{I}$ c c.c. saline solution. The short lines of the time marking show seconds, the long lines 5 seconds.

It would appear that rather too far-reaching deductions have been drawn from the action of atropine in cutting out the inhibitory control of the vagus. Since cutting the vagi always raises the blood pressure, while the administration of atropine always lowers it, in the $\operatorname{dog}$ at any rate; and since, on injection of atropine, the volume of both limb and intestinal wall always follow passively the blood pressure, we must conclude that atropine acts upon the heart in a manner quite 
different from that of section of the vagi. (Fig. 7.) It seems, in fact, that with atropine, although the vagus inhibition is removed, there is a much more powerful effect acting upon the circulation in an opposite sense, namely, a paralytic effect on the heart muscle itself.

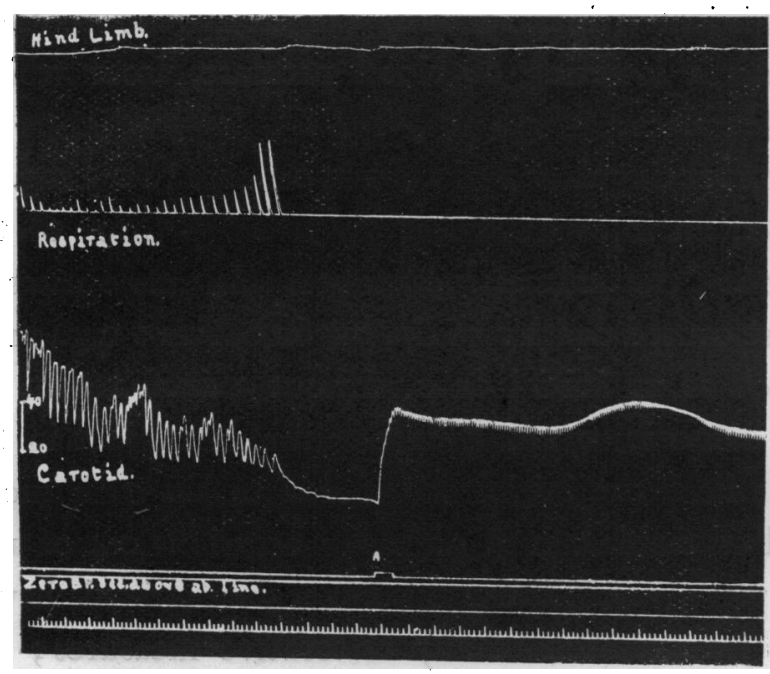

Figure 7.-Dog, $5.2 \mathrm{~kg}$. Overdose of chloroform. At A, both vagi were cut simultaneously; sudden rise of blood pressure occurs. Respiration, which had stopped, was not re-established, and blood pressure gradually falls again. The animal died in about 6 minutes.

\section{The Effects of Rapidly Repeated Doses of the Drugs}

It is well known that a very marked tolerance to atropine as well as to other drugs can be established both in animals and man by gradually increasing the dosage over a period of days, weeks, or months. It is, however, somewhat surprising to find that within the limits of time occupied by a single experiment a dog can be brought to withstand, manifesting only a comparatively slight reaction, a dose which, if administered at the beginning of the experiment, would have been certainly and quickly fatal; nay, further, in many cases even a large multiple of this. This rapid immunity or tolerance to the poisonous effects of a drug is not referred to in any of the books or papers to which I have had access, though the phenomenon is so striking that one can scarcely believe it has escaped the notice of pharmacologists. 
By commencing with small doses and gradually increasing their size, animals of 5 to $12 \mathrm{~kg}$. body weight have, in the course of an experiment lasting one and a half hours, been rendered so immune to the ill effects of the drug as to tolerate as large a quantity as 0.4 gramme injected intravenously (Fig. I) without other apparent ill effects than a lowering of blood pressure, from which recovery gradually takes place. ${ }^{1}$

The commencing dose varied from $0.1 \mathrm{mg}$. to $0.5 \mathrm{mg}$. of atropine, hyoscine, etc., an injection being given every three to ten minutes afterwards until the animal succumbed. In some cases $0.5 \mathrm{mg}$. as an initial dose caused death rapidly, but in others $1 \mathrm{mg}$., or even $2 \mathrm{mg}$., could be used as an initial dose without a fatal result, the animals showing the same idiosyncrasy as the human subject in this respect. In all cases where the initial dose failed to kill each successive dose was doubled or trebled until on many occasions 0.4 of a gramme of the alkaloid was given. "From this latter dose animals have recovered without the intervention of any restorative measures; double this a mount has been given, and the animal kept alive by artificial respiration, the blood pressure, which was very low after the injection, rising almost to the normal. But immediately on stoppage of the artificial respiration the pressure would fall and death ensue.

It does not make any difference which of the drugs, atropine, hyoscine, hyoscyamine, duboisine, daturine or scopolamine, are used to commence with in the process of obtaining this tolerance; any one can be used in increasing doses; then, when the dose has become large, an increased dose of any of the others given with no different result than would be obtained were the first drug continued-each drug immunising the animal from all the rest of the series. Further, the serum of an animal which has been rendered immune to large doses of these drugs will, if injected into another animal, confer an immunity on it.

Thus a first dose of $0.5 \mathrm{mg}$. of duboisine injected into a dog of $6 \mathrm{~kg}$. weight produced rapid fall of blood pressure and death. (See

1. It is impossible to make any definite statements as to the permanent effects of such a dose; as in this and all other cases the animal was killed under the anaesthetic. 
Fig. 8.) Later, blood serum, ${ }^{1}$ obtained from an animal which had previously had large doses of hyoscine and atropine, was injected into another $\operatorname{dog} 6 \mathrm{~kg}$. weight. The serum, amounting to 80 c.c., was given in eight doses by means of a Io c.c. syringe. A very slight effect was noticed with the first and second dose, given at intervals of three minutes, and between the second and third doses; the remaining six doses were given as quickly as the instrument could be filled, and no effect was observed. $12.5 \mathrm{mg}$. (twenty-five times the dose which killed the same weight of dog mentioned in Fig. 8) of duboisine were injected about ten minutes afterwards with practically no effect, as seen in Fig. 9.

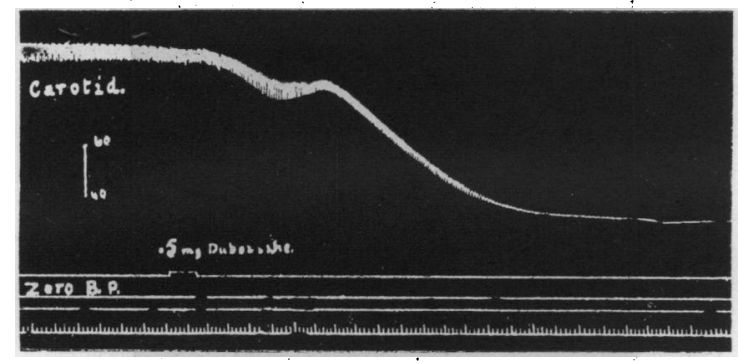

(FIGURE 8.-Dog, $6 \mathrm{~kg}$, Duboisine, $0.5 \mathrm{mg}$. caused death in two minutes. This was a primary dose.

From this experiment it would be rash to conclude that anything of the nature of an antitoxin had been formed. The first dose (Io c.c.) of serum from the drugged animal had the effect of cutting out the vagus action in the second animal, and it is probable that this serum contained therefore considerable quantities of the drug injected. This has an important bearing upon one theory of toleration, rendering it clear that since the serum contained the drug in an active form the tolerance established in the first dog could not be due to rapid elimination.

1. Blood was drawn from an animal which had had large quantities of hyoscine and atropine, and after standing twenty-four hours the serum was drawn off with a pipette in the usual manner. 


\section{Action on the Respiratory System}

It is well known that the first effect of atropine upon the respiration is to increase both the frequency and extent of the movements. (Figs. I, 5, 9.) Subsequently, however, the respiratory centre is paralysed. (Figs. 6 and 7.) I have been able to confirm the statement made by Reichert, ${ }^{1}$ and quoted by Sollmann, ${ }^{2}$ that an animal may recover from many times the minimal fatal dose if artificial respiration be maintained. This power of recovery on the part of the respiratory centre is of supreme practical importance in dealing with cases of poisoning by this drug.

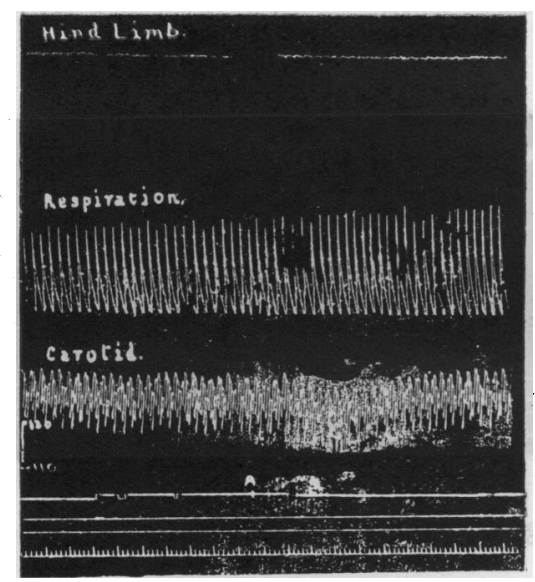

Figure 9.-Dog, $6 \mathrm{~kg}$. 80 c.c. of blood serum from a dog (which had had altogether $65 \mathrm{mg}$. of hyoscine and $\mathrm{I} \cdot 64$ grams of atropine in an experiment extending over nearly two hours) was administered. At A, $12.5 \mathrm{mg}$. of duboisine was administered with practically no effect. Previously (Fig. 8) $0.5 \mathrm{mg}$. ( $\frac{1}{25}$ the dose) given as a first injection, without a preliminary one of blood serum, killed a dog of the same weight in two minutes.

'When the blood pressure has been depressed by an overdose of chloroform, section of the two vagi, by cutting off the medullary effect, will release the heart; the beat will once again recover its normal character, and the blood pressure will bound up.'3 A natural

\footnotetext{
I. Reichert, Pbila. Med. Four., Jan. 19th, Ig0r.

2. Sollmann, op. cit.

3. Dixon, op. cit.
} 
inference would be that atropine, by cutting off the tonic effects - of the vagus, would have a similar effect. This, however, has not been the case in my experiments. ${ }^{1}$ In chloroform poisoning, just as in the normal condition, atropine does not raise the blood pressure, but lowers it. I have not found the slightest benefit to accrue when atropine has been administered to an animal whose circulation is depressed with chloroform or other anaesthetic.

The use of atropine prior to the administration of chloroform has been strongly advocated and no less strongly opposed by various writers, e.g., Brodie and Crouch, ${ }^{2} \mathrm{~J}_{\text {. Harley }}{ }^{3}$, Dastre ${ }^{4}, \mathrm{Pith}^{5} ; \mathrm{Fraser}^{6}$, Brown-Sequard7, Dastre and Morat ${ }^{8}$, Schäfer ${ }^{9}$, Schäfer and Scharlieb $^{10}$, Hewitt ${ }^{11}$, some of whom question the great danger of primary inhibition of the heart through excitation of the vagus, and also the benefit supposed to be derived from a preliminary dose of atropine.

The dose required to eliminate vagus action in dogs varies greatly per kg. of body weight in different individuals. This variability, we know, exists to as great an extent in the human subject. In view of the fact that we have no convenient means of testing vagus condition in the human subject, it follows that after administering a dose of atropine previously determined upon we are in the dark as to whether we have obtained the desired effect. Should we invariably give a large dose, disagreeable and possibly fatal results might ensue ; numerous cases have been recorded where small doses have produced ill effects.

I. I have not invariably found benefit from cutting the vagi, although often, as Dixon states, it restores blood pressure. In the case of the animal whose tracing is reproduced in Fig. 7 , although a sudden rise of blood pressure took place, respiration was not restored; the blood pressure again fell, and death rapidly ensued.

2. Trans. Soc. Anaestb., Vol. VI, pp. 70 and $81,1903$.

3. Brit. Med. Four., Vol. II, p. 320, 1868.

4. Soc. Biol., p. 242, 1883 .

5. Pitha, 186r. Quoted by Schäfer from Dastre.

6. Brit. Med. Four., Vol. II, p. 715, 1880 .

7. Brown-Sequard (C. r. Soc. Biol., p. 289, 1883).

8. Dastre and Motat (Lyon Med., 1882, and C. r. Soc. Biol., pp. 242 and 259, 1883).

9. Schäfer, Brit. Med. Four., Vol. II, p. 620, 1880.

10. Trans. Royal Soc. Edin.; p. 333, 1904.

11. Anaesthetics and their administration, pp. 230, 259, 503, 1907. 
Harley advocates 0.01 to 0.025 grain, and Dastre 0.0015 gramme (0.023 grain). These doses would certainly cause serious symptoms in some subjects, and would probably cause discomfort in nearly all, and in spite of their size we have no guarantee that the quantity is sufficient to abolish vagus action. It may also be argued that after an anaesthetic the sleepiness and depression are such that the dose of atropine would be eliminated before consciousness completely returned. This would only be the case after prolonged anaesthesia. After short operations, where the anaesthetic is skilfully administered, consciousness returns with comparative rapidity, while the effects of atropine last twelve to twenty-four hours; and in these short cases the atropine is quite as necessary as in cases of prolonged anaesthesia if, as stated, the danger to be chiefly averted is that of primary cardiac inhibition.

As regards myself, 0.01 grain administered hypodermically to test the effect on blood pressure produced very disagreeable results; headache, defective vision, dryness of the fauces, and a slight incoordination of the muscles, particularly of the lower limbs, being the prominent symptoms. Blood pressure which was taken previously for three consecutive days at the same hour with Janeway's instrument was recorded every five minutes after the injection at first, then later every ten minutes. It showed a gradual fall until it was reduced by $20 \mathrm{~mm}$. of mercury. Dr. - , who was treated in the same manner, but only took 0.0067 grain of atropine, had less pronounced symptoms, but still sufficient to cause considerable discomfort, and a very slight change of blood pressure, which was lowered by Io $\mathrm{mm}$. of mercury.

Among the more recent advocates of the use of atropine, Professor Schäfer must be specially mentioned. His method was to inject atropine prior to the administration of the anaesthetic, and hypodermically. In my experiments this has not been done. The atropine has been injected into a vein during the actual administration, and at various stages, and with the blood pressure at different levels. So far as my experiments go they do not support the view that the atropine is beneficial. 
So far as I can conclude from my experiments, adrenalin would be of distinctly more benefit, though, since its effect is transitory, as a rule frequent small doses must be administered in order to be of benefit. If the heart has not completely stopped, adrenalin causes more active contractions ${ }^{1}$ and consequent rise of blood pressure, which a few doses at short intervals may make permanent. If the heart has ceased absolutely, I have never obtained any result. This restoration of heart beat may be obtained after the heart movements can no longer be felt through the chest wall, but when, if the chest wall is opened, small movements can still be observed.

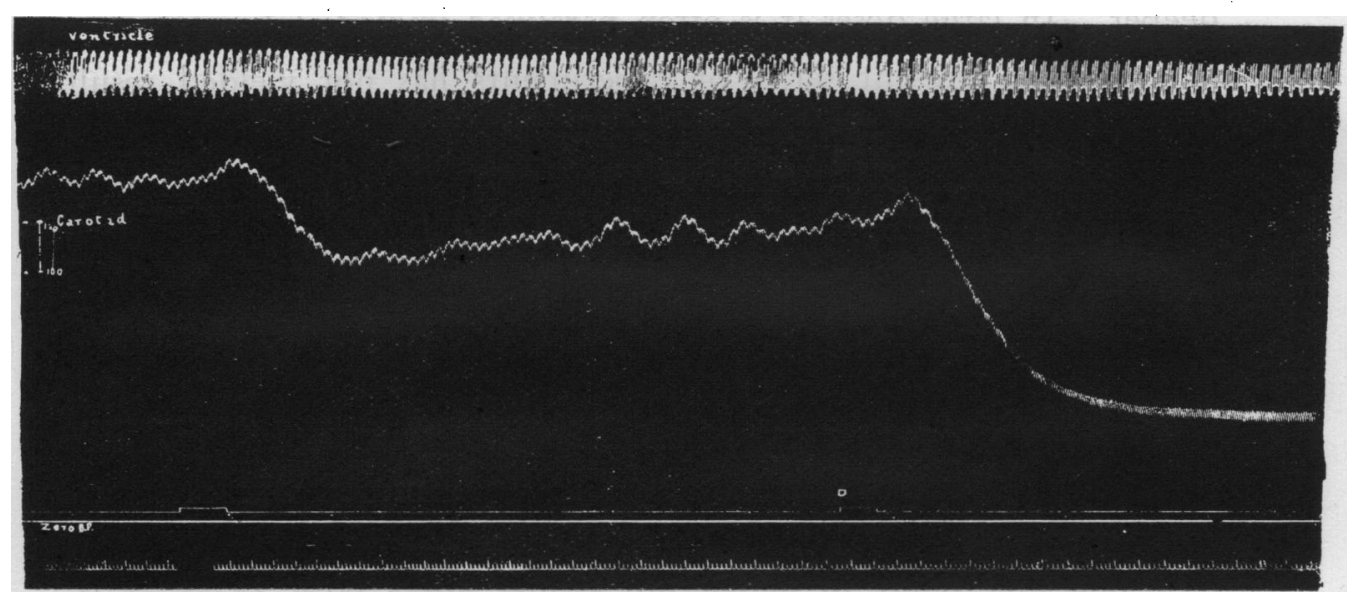

FIGURE 10.-Dog, $7 \cdot 2 \mathrm{~kg}$. Ether, atropine. At A, 0.02 gram causes, after a slight preliminary rise, a fall in blood pressure and slowing of heart with diminution of the force of the beats. At B, 0.08 of a gram was given with similar, but more pronounced. results.

\section{SUMMARY}

I. In dogs atropine, hyoscine, hyoscyamine, scopolamine, and daturine produce, whether in small or large doses, a lowering of the blood pressure.

2. The volume of a limb or portion of intestinal wall always becomes diminished concomitantly with the fall of blood pressure. of the body.

The rise with adrenalin is due also to stimulation of vasomotor nerve endings in various parts 
3. The conclusion seems justified that although these drugs eliminate the tonic inhibitory action of the vagus, they have a simultaneous action on the heart substance, diminishing the output. This paralytic effect upon the heart is shown also by direct experiment upon this organ.

4. By frequent administration of increasing doses of these drugs an animal may be brought into a condition of tolerance within one or two hours, so that at the end of this time it will withstand (with comparatively slight reaction) very many times the dose which would have been fatal at the beginning of the experiment.

5. In small doses the respiration is quickened and rendered deeper. In large doses it is often paralysed immediately.

6. The present series of experiments has not yielded results which would tend to encourage the use of atropine in chloroform poisoning. Adrenalin seems to be of much more, though limited, utility.

\section{Note added February 6th, 1908}

In consequence of some criticism of the preceding observations, which have been privately offered, I have performed a further series of experiments.

The first point in which my results differ from those of the majority of experimenters is that in the dog certainly, and probably also in other animals, a dose of atropine sufficient to produce any effect at all upon the blood pressure does not raise it, but invariably lowers it. This can only mean that the increased force and frequency of the heart's action, which must be brought about by the mere fact of eliminating vagus action, is counteracted even in minimal doses by another effect of atropine, viz., a depressant effect. In other words, the effect of the administration of atropine is something other than a simple cutting out of the vagus.

On this point I have performed a series of ten additional experiments on dogs, and find myself totally unable, under any circumstances whatever, to raise the blood pressure of the animal. The 
attempt has been made on a dog without any anaesthetic as well as under the influence of chloroform, ether, the A.C.E. mixture, morphia and curari, and further with the blood pressure at various levels. In all cases when the dose has been sufficient to produce any effect at all there has been a fall and not a rise of blood pressure. It is scarcely necessary to state that account has been taken only of the initial injection in any one animal, since it has never been supposed that atropine would raise the blood pressure after the vagus terminals have once been paralysed. In my experiments 0.5 c.c. of I-I0000 solution per $\mathrm{kg}$. of body weight has usually been found to be the smallest dose which will paralyse the vagus terminals, ${ }^{1}$ and this dose never raises the blood pressure.

The second point of interest in the above communication is that during the course of a single experiment lasting two or three hours a tolerance to the action of atropine may be established. It is not pretended that the large doses mentioned in the body of the paper would be completely recovered from, but that they produce effects which are exceedingly small as compared with effects which would be produced at the beginning of the experiment. This general result has been confirmed in ten fresh experiments.

I. The dose varies considerably in different animals, some requiring less, and some a greater dose. This, however, is about the average. 